$\alpha_{1}$-ANTITRYPSIN DEFICIENCY

\title{
Correlation between annual change in health status and computer tomography derived lung density in subjects with $\alpha_{1}$-antitrypsin deficiency
}

\author{
J Stolk, W H Ng, M E Bakker, J H C Reiber, K F Rabe, H Putter, B C Stoel
}

Thorax 2003;58:1027-1030

See end of article for authors' affiliations .....................

Correspondence to: Dr J Stolk, Department of Radiology and Division of Image Processing, Leiden University Medical Center, Leiden, The Netherlands; j.stolk.long@lumc.nl

Received 13 May 2003 Accepted

1 September 2003
Background: There is increasing recognition that questionnaires of health status and lung density measurements are more sensitive tools for assessing progression of emphysema than forced expiratory volume in 1 second $\left(\mathrm{FEV}_{1}\right)$ and transfer coefficient (KCO). A study was undertaken to investigate prospectively the correlation between annual change in health status and computer tomography (CT) derived lung density in subjects with $\alpha_{1}$-antitrypsin deficiency.

Methods: Twenty two patients of mean (SD) age 40.7 (9.2) years with ZZ type $\alpha_{1}$-antitrypsin deficiency were investigated at baseline and 30 months later by $\mathrm{FEV}_{1}$ and $\mathrm{KCO}$, St George Respiratory Questionnaire (SGRQ), and by a spiral CT scan of the chest. CT data of chest images were analysed using software designed for automated lung contour detection and lung density measurements. The density data were corrected for changes in inspiration levels.

Results: Changes in lung density, expressed as 15th percentile point or relative area below $-950 \mathrm{HU}$, correlated well with changes in health status (SGRQ total score): $R=-0.56, \mathrm{p}=0.007$ or $R=0.6, \mathrm{p}$ $=0.003$. Neither changes in health status nor changes in lung density correlated significantly with changes in $\mathrm{FEV}_{1}$ or changes in $\mathrm{KCO}$.

Conclusions: The SGRQ total score (which is a global measure in COPD) and lung density (a specific measure of emphysema) are sensitive to deterioration in patients with $\alpha_{1}$-antitrypsin deficiency. This finding may facilitate future studies with new drugs specific for emphysema, a frequently occurring component of COPD.
C hronic obstructive pulmonary disease (COPD) in individuals with $\alpha_{1}$-antitrypsin deficiency of the $\mathrm{Z}$ phenotype is frequently regarded as a classic example of a condition where genetic predisposition and environmental exposure interact. ${ }^{1}$ Emphysema is the most prevalent clinical phenotype of COPD in $\alpha_{1}$-antitrypsin deficiency and its progression is expressed in the literature as annual decline in forced expiratory volume in 1 second $\left(\mathrm{FEV}_{1}\right) .{ }^{2}$ Compared with non-deficient individuals, individuals with $\alpha_{1}$-antitrypsin deficiency who have never smoked have a relatively rapid annual decline in $\mathrm{FEV}_{1}$, with mean slopes reported between 47 and $86 \mathrm{ml} /$ year. $^{3}$

In some countries treatment by weekly infusions of human plasma derived $\alpha_{1}$-antitrypsin is available but, to date, no effect of $\alpha_{1}$-antitrypsin on annual decline in $\mathrm{FEV}_{1}$ has been proved in randomised trials. Power calculations, however, have shown that a large number of patients (550) for a long period of time ( 3 years) would be needed to show a statistically significant reduction of $50 \%$ in the annual decline in $\mathrm{FEV}_{1}{ }^{4}$ Such studies are almost impossible to organise and therefore more sensitive outcome parameters for trials with new drugs for patients with $\alpha_{1}$-antitrypsin deficiency need to be studied.

As an alternative to pulmonary function tests, measurement of health status is increasingly recognised as a tool for assessing treatment effects in clinical trials, both for asthma and COPD. ${ }^{5}$ The St George's Respiratory Questionnaire (SGRQ) was used in a 3 month study of salmeterol in COPD for assessment of treatment efficacy. ${ }^{6}$ Patients who scored the treatment as being effective and who had completed three questionnaires during the study had a mean improvement in SGRQ total score of 4.3 units (95\% CI 1.8 to 6.9). In the ISOLDE trial of fluticasone in patients with moderate to severe COPD the rate of decline in the SGRQ score was reduced by fluticasone by nearly $40 \% .^{7}$ Moreover, the difference between steroid and placebo treated groups widened progressively with time. This difference supported the registration of fluticasone propionate for COPD in the UK.

Lung densitometry measured by computed tomographic (CT) scanning is another new method for assessing treatment efficacy. ${ }^{8}$ In this technique emphysema can be quantified by two measures-relative area below -950 HU (RA-950) and 15th percentile point (Perc15). Both are derived from the frequency distribution (histogram) of the densities of all lung voxels. RA-950 is defined as the relative area (RA) under the curve of the histogram below a threshold of -950 Hounsfield Units (HU), and Percl5 is defined as the cut off density value in $\mathrm{HU}$ for which $15 \%$ of all voxels has a lower value.

In a landmark cross sectional clinical study, lung densitometry expressed as the 5 th percentile point (Perc5) showed a high correlation with both quantitative pathology scores of emphysema $(R=0.77, \mathrm{p}<0.001) .{ }^{9}$ In addition, cross sectional studies by Gevenois et $a l^{10}$ on the correlation between lung density (RA-950) and quantitative pathology showed a Pearson correlation of $0.70(\mathrm{p}<0.001)$.

CT lung densitometry has recently been used in longitudinal studies, demonstrating that lung densitometry is more sensitive than $\mathrm{FEV}_{1}$ in detecting the progression of emphysema. ${ }^{411}$ However, the annual decline in $\mathrm{FEV}_{1}$ did not correlate with the annual decline in density.

The positive results of SGRQ as an outcome parameter in studies such as the ISOLDE trial prompted us to assess the correlation between annual change in SGRQ total score and the annual decline in lung density in subjects with $\alpha_{1}$ antitrypsin deficiency of ZZ phenotype. 
Table 1 Baseline characteristics of study patients

\begin{tabular}{lll}
\hline & Absolute* & \% predicted \\
\hline Sex (M/F) & $10 / 12$ & - \\
Age (years) & $40.7(9.2)$ & - \\
Body mass index $\left(\mathrm{kg} / \mathrm{m}^{2}\right.$ ) & $25.2(5.0)$ & - \\
Inhaled steroids (yes/no) & $14 / 8$ & - \\
Smoking status (current/ex/never) & $5 / 14 / 3$ & \\
Smoking history (pack years) & $14.2(12)$ & \\
FEV (I) & $1.93(1.3)$ & $56.0(31.9)$ \\
KCO (mmol/min/kPa/l) & $1.03(0.4)$ & $61.4(23.6)$ \\
SGRQ total score & $32.4(20.1)$ & - \\
Lung density (15th percentile & $-927.9(44.2)$ & - \\
point) (HU) & & \\
\hline
\end{tabular}

*Mean (SD) values.

$\mathrm{FEV}_{1}=$ forced expiratory volume in 1 second; $\mathrm{KCO}=$ carbon monoxide transfer coefficient; SGRQ $=$ St George's Respiratory Questionnaire.

\section{METHODS}

Twenty two subjects with newly detected $\alpha_{1}$-antitrypsin deficiency of ZZ phenotype volunteered to participate in the study. The characteristics of the patients are shown in table 1 . None of them received $\alpha_{1}$-antitrypsin replacement therapy. Each patient was examined at baseline and after 30 months. If patients were free of exacerbations during the previous month, pulmonary function testing, spiral CT scanning of the chest, and a SGRQ were performed. The ethical board of LUMC approved the study and all patients gave informed consent.

Pre-bronchodilator spirometric tests were performed according to ERS guidelines with a rolling seal spirometer followed by post-bronchodilator spirometric tests after inhalation of $400 \mu \mathrm{g}$ salbutamol. ${ }^{12}$ Carbon monoxide transfer coefficient (KCO) was measured by single breath helium dilution on a Jaeger Masterscreen (Viasys, de Bilt, Netherlands). ${ }^{13}$

The SGRQ was applied as recommended ${ }^{14}$ with questions referring to the 3 months preceding the study visit.

Spiral CT scanning was performed as previously described using a Philips SR 7000 or Philips AVE scanner. ${ }^{4}$ Subjects inhaled $400 \mu \mathrm{g}$ salbutamol during the hour before the scan. All patients were scanned with instructions to inhale to full inspiration. At baseline and follow up, each patient was scanned on the same scanner. Reconstruction algorithms were kept the same over the study period.

Lung density was measured using software developed by us in cooperation with MEDIS Medical Imaging Systems BV, Leiden, the Netherlands. ${ }^{15}$ After automatic lung contour detection, the lung volume during breath holding was calculated followed by analysis of the density histogram of all lung voxels. The CT data were independently analysed by two observers (WH, EB) and the resulting measurements of lung volume and density were averaged. There was good agreement between the observers (mean (SD) difference in volume, Percl5, and RA-950 was -0.15 (2.1) $\mathrm{ml}, 0.27$ (1.5) $\mathrm{HU}$, and $0.43(0.58) \%$, respectively).

\section{Statistical analysis}

The density data were corrected for changes in inspiration levels between baseline and follow up using a linear mixed effects model fit by maximum likelihood, with log-transformed lung volume as random effect and log-transformed Perc15 or RA-950 as fixed effect. The derived slope between $\log$ volume and $\log$ Percl5 $(-1.49 \log (\mathrm{HU}) / \log (\mathrm{ml}))$ or RA$950(20.15 \% / \log (\mathrm{ml}))$ was used for correction of the data for each individual patient.

The correlation between changes in $\mathrm{FEV}_{1}, \mathrm{Kco}$, Percl5, and RA-950 and changes in SGRQ were assessed by the Spearman correlation coefficient as the changes were not normally distributed. In order to compare our baseline measurements with those in the literature, the correlation between all baseline measurements was also calculated.

\section{RESULTS}

\section{Patient demographic data}

At study entry six of the 22 patients had a pre-bronchodilator $\mathrm{FEV}_{1}$ and KCO above $80 \%$ predicted. The remaining 16 patients had a mean post-bronchodilator $\mathrm{FEV}_{1}$ of $38 \%$ predicted (range $25-54 \%$ predicted). Of these 16 patients, two could not perform a single breath helium dilution measurement for Kco; the remaining 14 had a mean Kco of $49 \%$ predicted (range $25-95 \%$ predicted).

\section{Correlation between baseline outcome parameters}

The baseline Percl5 correlated well with baseline KCO $(R=0.66, \mathrm{p}=0.001)$ and with $\mathrm{FEV}_{1}(R=0.68, \mathrm{p}=0.0001)$, which are comparable to values reported by Gould et al. ${ }^{9}$ Similar results were obtained with RA-950 (table 2). Baseline SGRQ total score correlated significantly with lung function and lung density measurements (table 3 ).

\section{Changes in health status and lung density}

The mean (SD) baseline SGRQ total score (32.4 (20.1)) correlated significantly with its annual change $(R=0.44$, $\mathrm{p}=0.041$, fig 1). The mean (95\% CI) change in SGRQ score during the study period was 6.5 units $(-2.9$ to 17.5). Baseline density measurements (Percl5 -928 (44.2) HU and RA-950 $12.2(11.6) \%$ ) did not significantly correlate with their annual change (Perc15: $R=-0.03, \mathrm{p}=0.89 ; \mathrm{RA}-950: \quad R=0.28$, $\mathrm{p}=0.21)$. The mean $(95 \% \mathrm{CI})$ change in Percl5 and RA-950 during the 30 month period was $-4.0 \mathrm{HU}(-26.0$ to 18.0$)$ and $3.4 \%$ ( -6.6 to 13.3$)$, respectively.

Changes in Perc15 correlated well with changes in health status as shown by the SGRQ total score $(R=-0.56$, $\mathrm{p}=0.007$, fig 2). Likewise, changes in RA-950 correlated with changes in the SGRQ total score $(R=0.604, \mathrm{p}=0.003$, fig 3). Changes in absolute values of $\mathrm{FEV}_{1}$ and Kco did not significantly correlate with changes in SGRQ or with changes in lung density (table 3 ).

\section{DISCUSSION}

We have found evidence that change in lung health status significantly correlates with change in lung density in patients with severe $\alpha_{1}$-antitrypsin deficiency. This analysis shows a relatively high correlation between the two measurements compared with a previously reported twofold lower correlation between decline in both SGRQ score and $\mathrm{FEV}_{1} \cdot{ }^{16}$ Our results suggest that a change in health status score caused by emphysema coincides with a change in a measure of lung pathology obtained by CT scanning. This suggests that lung density measurements may be a useful parameter for the evaluation of new drugs specifically designed for the treatment of emphysema.

To our knowledge, this is the first report to show that changes in health status and lung density are significantly

Table 2 Correlation between baseline lung function and baseline lung density

\begin{tabular}{llllll}
\hline & \multicolumn{2}{l}{ Baseline Perc15 } & & \multicolumn{2}{l}{ Baseline RA-950 } \\
\cline { 2 - 3 } \cline { 5 - 6 } \cline { 5 - 6 } & $\boldsymbol{R}$ & P value & & $\boldsymbol{R}$ & P value \\
\hline Baseline FEV 1 & $0.64^{*}$ & 0.001 & & $-0.65^{*}$ & 0.001 \\
Baseline KCO & $0.57^{*}$ & 0.007 & & $-0.60^{*}$ & 0.004 \\
\hline
\end{tabular}

*Correlation significant at 0.05 level (two tailed).

$R=$ Spearman correlation coefficient; Perc $15=15^{\text {th }}$ percentile point; RA-

$950=$ relative area below $-950 \mathrm{HU} \mathrm{FEV}_{1}=$ forced expiratory volume in

1 second; $\mathrm{KCO}=$ carbon monoxide transfer coefficient. 
Table 3 Correlation between St George Respiratory Questionnaire (SGRQ) and both lung function and lung density

\begin{tabular}{|c|c|c|c|c|c|c|c|c|}
\hline & \multicolumn{2}{|c|}{ Baseline $\mathrm{FEV}_{1}$} & \multicolumn{2}{|c|}{ Baseline Kco } & \multicolumn{2}{|c|}{ Baseline Perc15 } & \multicolumn{2}{|c|}{ Baseline RA-950 } \\
\hline & $R$ & $\mathrm{p}$ value & $R$ & p value & $R$ & p value & $R$ & p value \\
\hline $\begin{array}{l}\text { Baseline } \\
\text { SGRQ }\end{array}$ & $-0.61^{*}$ & 0.002 & $-0.54^{*}$ & 0.011 & $-0.47^{*}$ & 0.028 & $0.50^{*}$ & 0.018 \\
\hline $\begin{array}{l}\text { Change in } \\
\text { SGRQ }\end{array}$ & $\Delta \mathrm{FEV}_{1}$ & & $\Delta \mathrm{Kco}$ & & $\Delta$ Percl5 & & $\Delta$ RA-950 & \\
\hline Healthy $(n=6)$ & -0.20 & 0.70 & 0.23 & 0.65 & -0.41 & 0.42 & 0.06 & 0.91 \\
\hline $\begin{array}{l}\text { Emphysema } \\
(n=16)\end{array}$ & -0.22 & 0.42 & -0.26 & 0.34 & $-0.74^{*}$ & 0.001 & $0.67^{*}$ & 0.004 \\
\hline $\begin{array}{l}\text { Total group } \\
(n=22)\end{array}$ & -0.28 & 0.21 & -0.18 & 0.44 & $-0.56^{*}$ & 0.007 & $0.60^{*}$ & 0.003 \\
\hline
\end{tabular}

${ }^{*}$ Correlation significant at 0.05 level (two tailed).

$R=$ Spearman correlation coefficient; $\Delta=$ change in 30 months; Perc $15=15$ th percentile point; $R A-950=$ relative area below $-950 \mathrm{HU} ; \mathrm{FEV}_{1}=$ forced expiratory volume in 1 second; $\mathrm{KCO}=$ carbon monoxide transfer coefficient.

correlated. Dowson et al were unable to find a correlation between SGRQ total score and HRCT derived lung density in a similar study population. ${ }^{11}$ As the SGRQ total score did not decline over 24 months in their study, only a weak correlation between annual decline in the activity domain of the SGRQ and FEV 1 was observed in $47 \mathrm{Pi} \mathrm{Z}$ subjects over this time. ${ }^{11}$ The difference between the results of their study and ours can be explained by the longer period of observation, the more sensitive measurement of lung density (due to the correction for changes in inspiration level and the fact that the entire lung volume was scanned), and the wider range in baseline characteristics of the patients in our study.

The mean change in SGRQ total score in our patients with lung function values below $80 \%$ predicted was high ( 8.7 units (range -2.9 to 19.8)), underscoring the rapid decline in patients with $\alpha_{1}$-antitrypsin deficiency. The mean change in those with normal lung function was 0.6 units (range -2.9 to $5.5)$ which is comparable to previous findings. ${ }^{5}$ On average, COPD patients without $\alpha_{1}$-antitrypsin deficiency reach a significant level of deterioration of 4 units every 15 months, while age related worsening in the SGRQ score of 0.12 units per year is observed in healthy subjects without COPD. ${ }^{5} \mathrm{~A}$ change in total score of 4 units over any given period of time is therefore seen as a clinically important reduction in health status, leaving a change of less than 4 units difficult to interpret.

Figure I shows that in our study population baseline SGRQ total score had a linear correlation with its change over time, indicating that healthy subjects will only have a small change in total score whereas patients with COPD will deteriorate more rapidly if their baseline health status was poor. We are

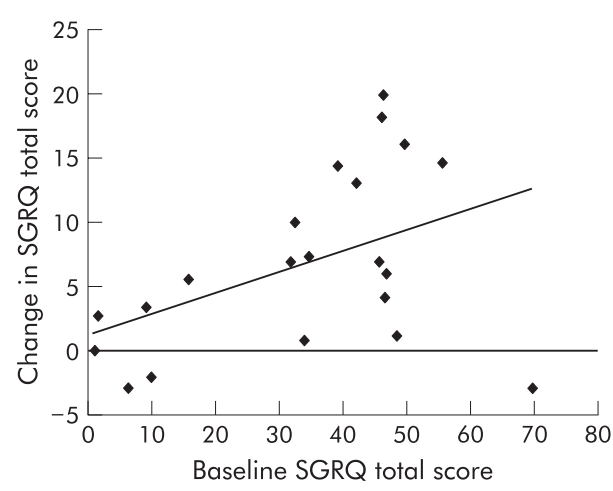

Figure 1 Relation between baseline SGRQ total score and its change over a mean period of 30 months in 22 patients with $\mathrm{Pi} Z \alpha_{1}$-antitrypsin deficiency (Spearman correlation coefficient $=0.44, p=0.04$ ). not aware of any studies in which the SGRQ total score was in the range of 85-100. In contrast, when $\mathrm{FEV}_{1}$ values are below $30 \%$ predicted, a small change over time is usually seen. ${ }^{17}$

Selection bias may have occurred in our study. However, recruitment of patients was based on consecutive referral with a diagnosis of $\alpha_{1}$-antitrypsin deficiency rather than reduced pulmonary function. Of the 22 patients, six were identified by family screening, five of which had a normal $\mathrm{FEV}_{1}$ and KCO. As can be seen in fig 2, the variation around the regression line was highest at low values of change in SGRQ score. All patients with normal lung function $(n=6)$ are clustered in this part of the graph (open circles). On the other hand, the change in their lung density was high in both a positive and a negative direction. No cross sectional or longitudinal data are available to enable interpretation of the change in lung density in a normal population.

We wish to emphasise the effect on the correlation between change in SGRQ total score and change in Percl5 of omitting the six patients with normal lung function (fig 2). This results in a significant improvement in correlation from 0.56 to $0.74(\mathrm{p}=0.001$, table 3$)$. No such improvement was found for change in RA-950 ( $R$ changed from 0.60 to 0.67 ). The different behaviour of Perc15 and RA-950 suggests that the percentile method is able to detect changes in mild emphysema, whereas the relative method is not. We therefore recommend reporting change in lung density by the percentile method. The authors are partners in the Spread

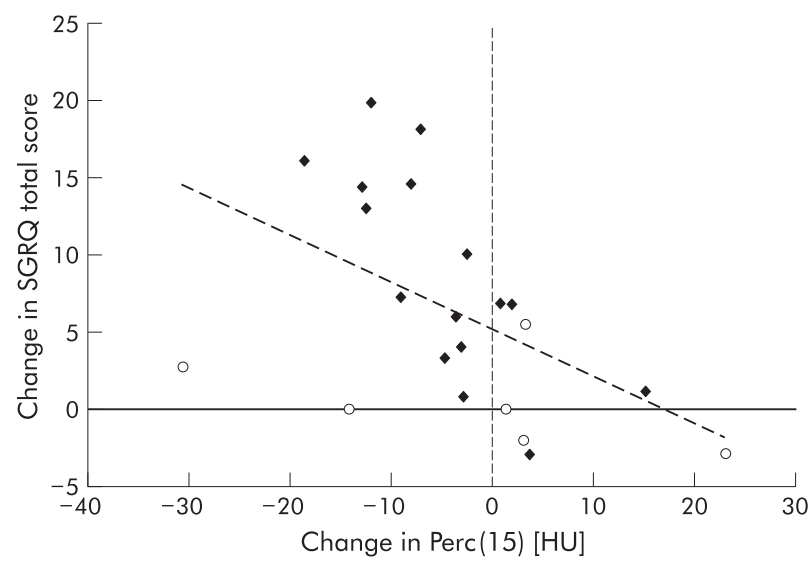

Figure 2 Relation between change in Perc 15 and change in SGRQ total score over a mean period of 30 months in 22 patients with $\mathrm{Pi} Z \alpha_{1}$ antitrypsin deficiency (Spearman correlation coefficient $=-0.56$, $p=0.007$ ). Open circles are patients with normal lung function. 


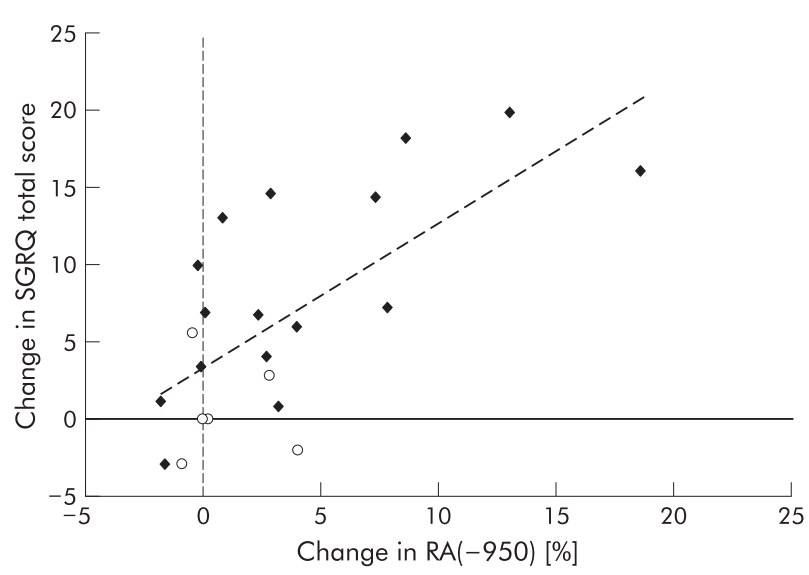

Figure 3 Relation between change in RA-950 and change in SGRQ total score over a mean period of 30 months in 22 patients with $\mathrm{Pi} Z \alpha_{1}$ antitrypsin deficiency (Spearman correlation coefficient $=0.60$, $p=0.003)$. Open circles are patients with normal lung function.

study which aims to study this aspect in more detail in a longitudinal multicentre study.

Although baseline values of $\mathrm{FEV}_{1}$ and Kco correlated well with baseline lung density and baseline SGRQ total score, no such correlation was found when the change in each of these parameters was calculated. This probably reflects either the low number of patients studied or the relatively short period of time.

While pulmonary function tests have been used for many years for monitoring the progression of emphysema, measuring lung density is a novel concept. This concept originates from cross sectional work which showed acceptable correlations between quantitative microscopic emphysema scores and both CT lung density values and KCO. ${ }^{10}$ In addition, previous studies have shown that air trapping is of no concern as scans are taken during inspiration. ${ }^{9}{ }^{10}$ However, since the level of inspiration by patients during follow up scans will never be precisely the same, corrections for inspiration level must be computed as indicated in our methods of analysis.

We conclude that the SGRQ total score, a global measure in COPD clinical assessment, and lung density, a specific measure of emphysema, are sensitive to deterioration in patients with $\alpha_{1}$-antitrypsin deficiency. It is hoped that this finding will encourage the development of trials for new drugs targeted at the treatment of emphysema, a frequently occurring component of COPD.

\section{ACKNOWLEDGEMENTS}

The authors thank their colleagues at the Alpha 1 International Registry (AIR, www.aatregistry.org) for valuable discussions. The Spread Project is described at www.lumc.nl/lkeb/spread.

\section{Authors' affiliations}

J Stolk, W H Ng, K F Rabe, Department of Radiology and Division of Image Processing, Leiden University Medical Center, Leiden, The Netherlands

M E Bakker, J H C Reiber, B C Stoel, Department of Radiology, Leiden University Medical Center, Leiden, The Netherlands

H Putter, Department of Medical Statistics, Leiden University Medical

Center, Leiden, The Netherlands

\section{REFERENCES}

1 Eriksson S. Pulmonary emphysema and alpha-1-antitrypsin deficiency. Acta Med Scand 1964:175:197-205.

2 Piitulainen E, Eriksson S. Decline in FEV1 related to smoking status in individuals with severe alpha-1-antitrypsin deficiency (Pi ZZ). Eur Respir J 1999; 13:247-51

3 Black LF, Kueppers F. Alpha-1-antitrypsin deficiency in non-smokers. Am Rev Respir Dis 1978:117:421-8.

4 Dirksen A, Dijkman JH, Madsen F, et al. A randomised clinical trial of alpha(1)-antitrypsin augmentation therapy. Am J Respir Crit Care Med 1999;160:1468-72.

5 Jones PW. Interpreting thresholds for a clinically significant change in health status in asthma and COPD. Eur Respir J 2002;19:398-404.

6 Jones PW, Bosh TK. Quality of life changes in COPD patients treated with salmeterol. Am J Respir Crit Care Med 1997;155:1283-9.

7 Burge PS, Calverley PMA, Jones PW, et al. Randomised, double blind, placebo controlled study of fluticasone propionate in patients with moderate to severe chronic obstructive pulmonary disease: the ISOLDE trial. BMJ 2000;320: 1297-303

8 Stockley RA. Alpha-1-antitrypsin deficiency: what next? Thorax 2001;55:614-8.

9 Gould GA, MacNee WA, McLean A, et al. CT measurements of lung density in life can quantitate distal airspace enlargements: an essential defining feature of human emphysema. Am Rev Respir Dis 1988;137:380-92.

10 Gevenois PA, De Vuyst P, de Maertelaer V, et al. Comparison of computed density and macroscopic morphometry in pulmonary emphysema. Am J Respir Crit Care Med 1996;154:187-92.

11 Dowson LJ, Guest PJ, Stockley RA. Longitudinal changes in physiological, radiological, and health status measurements in alpha(1)-antitrypsin deficiency and factors associated with decline. Am J Respir Crit Care Med 2001;164:1805-9.

12 European Respiratory Society. Standardized lung function testing. Lung volumes and forced ventilatory flows: 1993 update. Eur Respir J 1993;6/Suppl 16):5-40.

13 Cotes JE, Chinn DJ, Quanjer, et al. Standardization of the measurement of transfer factor (diffusion capacity). Eur Respir J 1993:48:119-24.

14 Jones PW, Quirk FH, Baveystock CM, et al. A self-complete measure for chronic airflow limitation: the St George's Respiratory Questionnaire. Am Rev Respir Dis 1992;145:1321-7.

15 Stoel BC, Vrooman HA, Stolk J, et al. Sources of error in lung densitometry with CT. Invest Radiol 1999:34:303-9.

16 Spencer S, Calverley PMA, Burge PS, et al. Health status deterioration in patients with chronic obstructive pulmonary disease. Am J Respir Crit Care Med 2001; 163:122-8.

17 Fletcher CM, Peto R. The natural history of chronic airflow obstruction. BMJ 1978;1:1645-8. 


\section{PostScript}

\section{LETTERS TO THE EDITOR}

If you have a burning desire to respond to a paper published in Thorax, why not make use of our "rapid response" option?

Log on to our website (www.thoraxinl. com), find the paper that interests you, and send your response via email by clicking on the "eletters" option in the box at the top right hand corner

Providing it isn't libellous or obscene, it will be posted within seven days. You can retrieve it by clicking on "read eletters" on our homepage.

The editors will decide as before whether to also publish it in a future paper issue.

\section{Squawks in pneumonia}

Squawks are short inspiratory wheezes that have been described in hypersensitivity pneumonitis and other fibrotic disorders. Little attention has been paid to the fact that they also occur in patients with pneumonia. In the course of studying the correlation of automated lung sound analysis with disease states in patients at a community teaching hospital, we noticed that squawks appeared to be more common in patients with pneumonia than we expected. We therefore examined the occurrence of squawks more systematically in 500 subjects who had been examined with a multichannel lung sound analyser (Stethographics Model STG-1602), as previously described.'

Seventy eight of the subjects in this population had a clinical diagnosis of pneumonia. All participants had been asked to breathe more deeply than normal with their mouths open. Two 20 second samples were taken. The Institutional Review Board of the Faulkner Hospital approved the study. Two experienced observers, blinded to the clinical diagnosis, used playback and waveform displays to identify squawks. They were defined according to the criteria initially described by Earis et al and adopted by CORSA. ${ }^{23}$ All channels from each subject were replayed and the waveforms of the data in the time domain were simultaneously examined. Only those sounds which fit both the auditory and waveform characteristics were considered to be squawks for the purposes of this study.

Squawks were present in 12 of the 78 patients with pneumonia and in none of 224 patients considered to have no significant lung disease. They were found in four of 18 patients with interstitial pulmonary fibrosis,
A

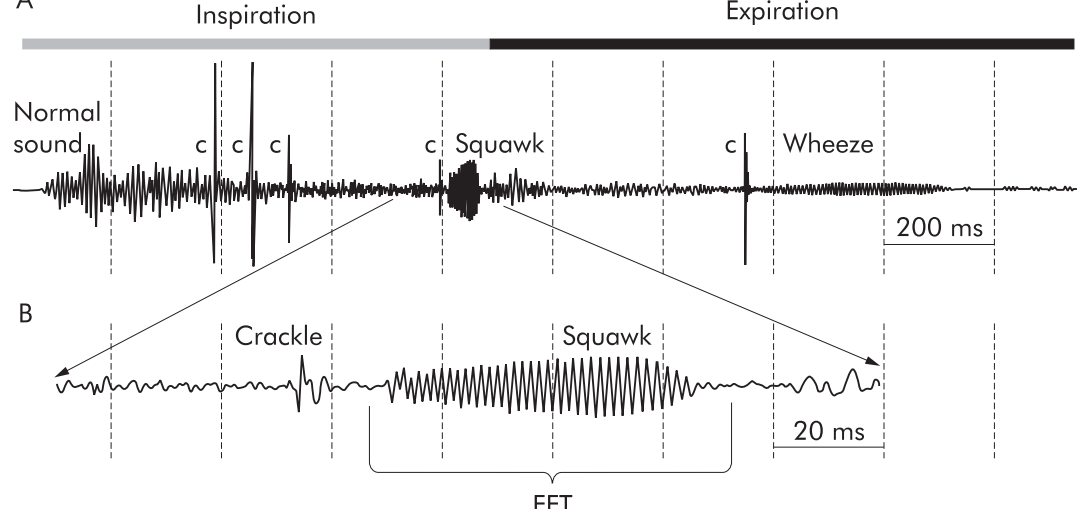

C

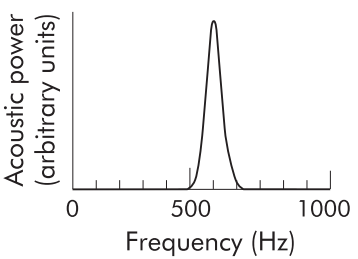

Figure 1 Time-amplitude plot of a sound recorded at the lung bases posteriorly (channel 14) in a patient with pneumonia. Waveforms are presented in both the unexpanded (A) and expanded (B) modes. (A) The unexpanded waveform shows one full breath. The solid bars above the unexpanded wave mark the respiratory cycle (the light bar indicates inspiration and the dark bar indicates expiration). The normal inspiratory sound can be seen as having almost random waveform fluctuations. Fine crackles (c) look like spikes on an unexpanded waveform. A squawk is present at the end of inspiration. One fine crackle and a wheeze are present during expiration. (B) The expanded squawk waveform exhibits monophonic sinusoidal fluctuations lasting approximately $60 \mathrm{~ms}$. (C) The squawk sound in the frequency domain shows a single peak at $600 \mathrm{~Hz}$. In this patient similar squawks appeared in three consecutive breaths during $20 \mathrm{~s}$ of deeper than normal breathing. They occurred approximately in the same location on the chest and at the same time in the respiratory cycle. These observations were typical of the squawks detected in our study. $\mathrm{FFT}=$ fast fourier transform. two of 41 patients with bronchial asthma, one of 79 patients with COPD, and in none of 56 patients with congestive heart failure. We also noted squawks in a patient with radiation pneumonitis and in one of the two patients in our database with hypersensitivity pneumonitis.

In nine of the 12 patients with pneumonia the squawks were in the same location as the radiological opacifications. In one patient the squawk was in a different location and in another the chest radiograph did not show evidence consistent with pneumonia until 4 days after the squawk was detected. In the remaining patient a squawk was heard over the left posterior mid chest. The portable chest radiograph was interpreted as technically suboptimal due to the patient's body habitus. The clinicians caring for this patient made a diagnosis of pneumonia and treated him accordingly.

Interestingly, one patient who clearly had congestive heart failure on a number of occasions had a squawk when we examined him. On re-examining his record he also had a diagnosis of pneumonia on that day. Similarly, there were two patients with squawks (one with COPD and one with asthma) who, on the day that the squawks were noted, had acute febrile illnesses consistent with pneumonia. In one patient the presence of a squawk led to reinterpretation of the chest radiograph as showing an area of opacification consistent with pneumonia.

The squawks in this study all had a distinctive sound that is readily distinguished from crackles, rhonchi, rubs, and most wheezing noises. Occasionally wheezes can be short and have a similar sound, but this occurs rarely as an isolated finding in inspiration. The squawks in this study all had sinusoidal waveforms as illustrated in the time-amplitude plot shown in fig 1 . The mean (SD) duration of these sounds was 64 (49) ms (range 16-228) and the mean (SD) frequency was 425 (110) Hz (range 200-667). These findings are similar to those of Earis et al. ${ }^{3}$

When a squawk is accurately identified, the question arises-what does it mean? In a patient who is not acutely ill, investigations to rule out hypersensitivity pneumonitis and the other fibrotic conditions mentioned above should be considered. In a patient with a clinical picture consistent with pneumonia, the presence of a squawk offers some objective evidence to support the diagnosis. It would seem reasonable to suggest that the patient should be followed up to be sure that the squawk disappears when the acute illness resolves to exclude the possibility that the acute illness was the mode of presentation of a chronic pulmonary disorder. Squawks can be helpful in providing evidence for pneumonia in lung areas where radiological visualisation may be suboptimal, such as below the dome of the diaphragm or in the retrocardiac region.

In summary, short inspiratory wheeze-like sounds are found in pneumonia. Other conditions that can cause them are chronic restrictive disorders but these are relatively uncommon compared with pneumonia. When there is no evidence of these restrictive disorders and an acute syndrome consistent 
with respiratory infection is present, squawks can provide relatively specific-although not very sensitive-evidence of pneumonia.

R Paciej, A Vyshedskiy, D Bana, R Murphy Faulkner/Brigham and Women Hospitals, Boston, MA, USA

Correspondence to: Dr R Murphy, 1153 Centre St, Suite 4990, Boston, MA 02130, USA; rmurphy@ faulknerhospital.org

Supported in part by grants from NIH SBIR (1R43HL70480-01) and from Stethographics Inc.

\section{References}

1 Bergstresser T, Ofengeim D, Vyshedskiy A, et al Sound transmission in the lung as a function of lung volume. J Appl Physiol 2002;93:667-74.

2 Earis JE, Marsh K, Pearson MG. The inspiratory "squawk" in extrinsic allergic alveolitis and other pulmonary fibroses. Thorax 1979;37:923-6.

3 Sovijarvi AHA, Vanderschoot J, Earis JE. Computerized respiratory sound analysis (CORSA): recommended standards for terms and techniques. Eur Respir Rev 2000; 10:585-649.

\section{BTS guidelines for the management of pleural infection}

We have read the BTS guidelines for the management of pleural infection ${ }^{1}$ and are concerned about the proposed antibiotic choices for the initial treatment of culture negative or pending pleural infection. Section 2.8 of the text and table 2 detail the antibiotic choices but, in our opinion, leave considerable gaps in antibacterial cover against the likely pathogens. In particular:

- Amoxycillin (text) is not reliably active against Staphylococcus aureus.

- Clindamycin has no activity against Gram negative aerobic organisms (especially not Haemophilus influenzae as mentioned in the text).

- Benzyl penicillin (table) rarely now has activity against Staphylococcus aureus and we suggest that relying on ciprofloxacin is unwise. In addition, this combination will not cover many anaerobic bacteria.

- We do not consider chloramphenicol is an appropriate agent to use in this category of patients in view of the serious side effect profile.

- Third generation cephalosporins such as ceftazidime and cefotaxime have unreliable activity against many anaerobic bacteria.

- Pneumococci are considerably less susceptible to ceftazidime than to other cephalosporins and penicillins ${ }^{2}$; the policy (table), however, suggests its use as a single agent.

- Piperacillin (text) is no longer available in the UK except in combination with a $\beta$ lactamase inhibitor.

We suggest that the antibiotic choices in the BTS guidelines for the management of pleural infection should be changed to the following:

- For community acquired pleural infection, either cefuroxime + metronidazole or $\mathrm{CO}-$ amoxiclav or (for the penicillin/cephalosporin allergic individuals) clindamycin + ciprofloxacin, all administered intravenously. Oral treatment choices would be co-amoxiclav or clindamycin + ciprofloxacin.
- For hospital acquired infection, clinicians should seek guidance from the local microbiologists but the following choices would be reasonable in the interim: piperacillin + tazobactam or cefotaxime/ceftriaxone + metronidazole or meropenem.

Relating to the initial diagnosis of pleural infections, we were also concerned that mycobacteria were mentioned only once in the article. Pleural fluid has a poor yield for diagnosis of tuberculosis and more emphasis should have been placed on the routine use of pleural biopsy for histology and culture of tuberculosis which has much higher diagnostic rates. The algorithm should include the investigation of pleural tuberculosis.

In conclusion, we would commend the inclusion of a medical microbiologist in discussions leading to guidelines dealing with the diagnosis and treatment of infections.

H S A Roberts*

Consultant Microbiologist, Princess Royal University Hospital, Farnborough Common, Orpington, Ken BR6 8ND, UK honor.roberts@bromleyhospitals.nhs.uk

*On behalf of the Outer South East London Microbiology Group: M Viagappan, University Hospital, Lewisham; A Mackay, Queen Elizabet Hospital, Woolwich; A Teall, Queen Elizabeth Hospital, Woolwich; H Roberts, Princess Royal University Hospital, Bromley; J Kensit, Queen Mary's Hospital, Sidcup; A Fowler, Queen Elizabeth Hospital Woolwich; A Shaw, Darent Valley Hospital.

\section{References}

1 Davies CWH, Gleeson FV, Davies RJO, et al. BTS guidelines for the management of pleural infection. Thorax 2003:58(Suppl II):ii1 8-28.

2 Spangler SK, Jacobs MR, Appelbaum PC Susceptibilities of 177 penicillin-susceptible and resistant pneumococci to FK 037, cefpirome, cefepime, ceftriaxone, cefotaxime, ceftazidime, imipenem, biapenem, meropenem, and vancomycin. Antimicrob Agents Chemother 1994;38:898-900.

3 Ferrer J. Pleural tuberculosis. Eur Respir J $1997 ; 10: 942-7$

\section{Authors' reply}

We would like to thank Dr Roberts and colleagues for their interest in the BTS guidelines on pleural infection ${ }^{1}$ and for their thoughtful letter. In our view, guidelines (particularly the first set in an area) exist partly to stimulate a debate which subsequently better informs care.

Since the advisory regimens in the guidelines were first drafted (and they are "advisory" and to be used in line with local microbiological advice), the microbiology of pleural infection has been greatly clarified (not least by the joint BTS/MRC trial in pleural infection). This same work is also identifying high risk patient groups, clarifying drain type choice, and accurately defining intrapleural treatment. We have no doubt that these new data, as well as some of the points raised by Dr Roberts and colleagues, will strengthen the next revision of the BTS guidelines. The recent data show that only $10 \%$ of community acquired infections are staphylococcal, while $50 \%$ of hospita acquired infections are due to staphylococcal disease and $66 \%$ of these are MRSA infections. Thus, a regimen with limited staphylococcal cover may be appropriate in community infection (although thorough anaerobic cover is needed here), but isolated meropenem in hospital acquired infection (suggested by Roberts and colleagues) would be ineffective for $25 \%$ of patients in this setting. Here we might currently favour vancomycin + meropenem (or similar). The BTS/MRC trial suggests that about $50 \%$ of patients with hospital acquired pleural infection are currently receiving ineffective empirical antibiotics-emphasising the importance of clarifying this issue. The suggestion of a combination of clindamycin + ciprofloxacin in community acquired infection seems a particularly elegant improvement on the regimen of clindamycin alone advocated in some US centres and mirrored in our original suggestions.

We share the view that, when the pleural infection guidelines are next updated, a microbiologist should be on the drafting panel and not only included during peer review. The drafting panel for these guidelines was a compromise between size and inclusivity in all the therapeutic areas, since it had to cover all the pleural syndromes. This, for example, led to the absence of an oncologist for the malignant effusion guideline and a physician with particular skills in cystic fibrosis for the pneumothorax guideline (again peer review was the chosen method for including these specialists). On the plus side, this led to an efficient guideline generation process. We have previously encouraged the BTS to constitute separate groups for each of the guidelines as they come up for future review for just this reason.

R J O Davies, C W H Davies, F V Gleeson Churchill Hospital, Headington, Oxford, UK

Correspondence to: Dr R J O Davies, Churchil Hospital, Headington, Oxford OX3 7ป, UK robert.davies@ndm.ox.ac.uk

\section{Reference}

1 Davies CWH, Gleeson FV, Davies RJO, et al. BTS guidelines for the management of pleural infection. Thorax 2003;58(Suppl II):ii 18-28.

\section{Bronchodilator reversibility testing in COPD}

In their paper on bronchodilator reversibility testing in COPD, Calverley and colleagues come to the intuitively sensible conclusion that, in severe COPD, bronchodilator responsiveness is a continuous variable. However, this conclusion is based on an analysis in which the change in forced expiratory volume in l second $\left(\mathrm{FEV}_{1}\right)$ effected by inhalation of a bronchodilator aerosol is related to the baseline (that is, initial) level As a result, the reported bronchodilator responses are subject to the error that can result from regression to the mean. ${ }^{2}$ The error can be minimised by relating the change to the mean level instead of the initial level, and it would be reassuring to see the data expressed in this form.

J E Cotes

Cell and Molecular Biosciences, Medical School Newcastle upon Tyne, UK; coterie@globalnet.co.uk

\section{References}

1 Calverley PMA, Burge PS, Spencer S, et al. Bronchodilator reversibility testing in chronic obstructive pulmonary disease. Thorax 2003;58:659-64. 
2 Oldham PD. A note on the analysis of repeated measurements of the same subjects. J Chronic Dis 1962; 15:969-77

\section{Authors' reply}

We thank Dr Cotes for his interest in our work $^{1}$ and his thoughtful comments. We did not include the suggested analysis as our purpose was to address the utility of a single bronchodilator reversibility test which is the way this is applied in routine practice. We agree that regression to the mean is a real possibility, but also believe that physiological variation in baseline airway calibre contributes to the "noise" in this particular signal. Unfortunately the number of times this test must be undertaken to minimise these confounding effects is not clear, but is likely to be too large to be possible in most clinics. Hence our caution about interpreting the significance of small changes in spirometry recorded on one visit.

P M A Calverley

Department of Medicine, Clinical Sciences Centre, University Hospital, Aintree, Liverpool L9 7AL, UK pmacal@liverpool.ac.uk

\section{Reference}

Calverley PMA, Burge PS, Spencer S, et al. Bronchodilator reversibility testing in chronic obstructive pulmonary disease. Thorax 2003:58:659-64.

\section{Prednisolone response in patients with COPD}

Our interpretation of the important data on corticosteroid responsiveness in chronic obstructive pulmonary disease (COPD) presented by Burge et al $^{1}$ is quite different from that of the authors. They have shown that those with an improvement in forced expiratory volume in 1 second $\left(\mathrm{FEV}_{1}\right)$ of $>20 \%$ following short term treatment with prednisolone had a more significant reduction in exacerbation frequency with longer term treatment with inhaled fluticasone than those without. Thus, arguably, the only important positive clinical outcome of long term treatment of COPD with inhaled corticosteroids is largely confined to those who are identified by a positive response to prednisolone. This is a powerful validation of the suggestion in current guidelines ${ }^{2}$ that decisions on the use of long term inhaled corticosteroid therapy are based on a careful assessment of the response to short term treatment with prednisolone.

Clinicians will continue to use short term prednisolone trials in patients with unclassified, apparently fixed, airflow obstruction since this is the only practical way to identify the minority with markedly corticosteroid responsive disease. The data presented by Burge et al are important in that they show that smaller responses to prednisolone can provide additional useful clinical guidance. They also suggest that the mechanism of the bronchodilator response to prednisolone might have features in common with the mechanism of inhibition of COPD exacerbations with corticosteroids. We have recently shown that the improvement in $\mathrm{FEV}_{1}$ and quality of life scores seen with prednisolone compared with placebo in COPD increases progressively with increasing eosinophilic airway inflammation, and is associated with amelioration in eosinophilic airway inflammation as reflected by a sixfold reduction in the induced sputum eosinophil count. Future studies should investigate whether treatment targeting eosinophilic airway inflammation in COPD results in a reduction in exacerbations, as it has clearly been shown to do in asthma. ${ }^{4}$

I D Pavord, R Siva, C E Brightling Institute for Lung Health, Glenfield Hospital, Leicester,

Correspondence to: Dr I D Pavord, Institute for Lung Health, Glenfield Hospital, Leicester, UK ian.pavord@uhl-tr.nhs.uk

\section{References}

1 Burge PS, Calverley PMA, Jones PW, et al. Prednisolone response in patients with chronic obstructive pulmonary disease: results from the ISOLDE study. Thorax 2003;58:654-8.

2 British Thoracic Society. Guidelines for the management of chronic obstructive pulmonary disease. Thorax 1997:52(Suppl 5):S1-28.

3 Brightling CE, Monterio W, Ward R, et al. Sputum eosinophilia and the short-term response to prednisolone in chronic obstructive pulmonary disease: a randomised controlled trial. Lancet 2000;356: 1480-5.

4 Green RH, Brightling CE, McKenna S, et al. Asthma exacerbations and sputum eosinophil counts. A randomised controlled trial. Lancet 2002:360:1715-23.

\section{Authors' reply}

We thank Dr Pavord and colleagues for their letter. ${ }^{1}$ We were asked to add the section relating the effect of prednisolone response to fluticasone related exacerbation prevention by the papers' referees. We originally omitted this as there was no relationship between the effect of exacerbation reduction and prednisolone response when prednisolone response was expressed as a continuous variable $(\mathrm{p}=0.84)$, and we were worried that the analysis based on responders and nonresponders would result in misinterpretation, which unfortunately has been the case. The main conclusion from the paper is that any separation of the patients into responder and non- responder groups is statistically flawed. The response is unimodally distributed and individual changes between two visits (that is, before and after prednisolone) suffer from the regression to the mean effect. We showed good evidence that this was in fact occurring, with "responders" declining by a mean $127 \mathrm{ml}$ in the 4 weeks before prednisolone, while those in whom forced expiratory volume in 1 second $\left(\mathrm{FEV}_{1}\right)$ was reduced by $>20 \%$ after prednisolone had a $47 \mathrm{ml}$ increase in $\mathrm{FEV}_{1}$ in the preceding 4 weeks. The most likely explanation for the spurious relationship between exacerbation reduction and prednisolone response related to confounding by a low $\mathrm{FEV}_{1}$. Responders were classified as those with a $20 \%$ increase in $\mathrm{FEV}_{1}$ as a percentage of the starting value favouring a "response" in those with the lowest $\mathrm{FEV}_{1}$ (a change from 0.8 to $0.96 \mathrm{l}$ in the lowest group) and exacerbations are also more frequent in those with a lower $\mathrm{FEV}_{1}$. A clinical decision made on a steroid trial is not a reliable, reproducible, or valid way of separating out "responders" and so cannot be recommended.

P S Burge

Birmingham Heartlands Hospital, Bordesley Green East, Birmingham, UK
P M A Calverley

University Hospital, Aintree, Liverpool, UK

P W Jones, S Spencer

St George's Hospital Medical School, London, UK

Correspondence to: Professor P S Burge, Birmingham Heartlands Hospital, Bordesley Green East Birmingham B9 5ST, UK Sherwood.burge@heartsol.wmids.nhs.uk

\section{Reference}

1 Burge PS, Calverley PMA, Jones PW, et al Prednisolone response in patients with chronic obstructive pulmonary disease: results from the ISOLDE study. Thorax 2003;58:654-8.

\section{Breathing exercises in asthma}

It was a pleasure to read a report of a well conducted trial into a complementary treatment for asthma. ${ }^{1}$ This is a subject which attracts considerable media attention. The authors of this report and the author of an accompanying editorial ${ }^{2}$ both introduce their articles with a reference to "a third of respondents in a recent asthma survey having tried one or more breathing techniques to relieve symptoms". ${ }^{3}$ While their quote is correct, the report to which they refer is of a survey of members of the UK National Asthma Campaign. Such a membership may not be typical of those with asthma. In a more recent study of a stratified cross section of the asthma population we found only $6 \%$ of the study population to be current users of complementary therapies. That use was greatest among those who expressed most concern regarding their current medication. ${ }^{4}$

M R Partridge

Imperial College London, NHLI, Charing Cross Hospital Campus, London W6 8RP, UK m.partridge@imperial.ac.uk

\section{References}

1 Cooper S, Oborne J, Newton S, et al. Effect of two breathing exercises (Buteyko and pranayama) in asthma: a randomised controlled trial. Thorax 2003:58:674-9

2 Thomas $M$. Breathing exercises and asthma. Thorax 2003;58:649-50.

3 Ernst E. Complementary therapies for asthma: what patients use. J Asthma 1998;35:667-71.

4 Partridge MR, Dockrell M, Smith NM. The use of complementary medicines by those with asthma. Respir Med 2003;97:436-8.

\section{CORRECTION}

\section{$\alpha_{1}$-Antitrypsin deficiency: paper by Stolk et al}

In the paper entitled "Correlation between annual change in health status and computer tomography derived lung density in subjects with $\alpha_{1}$-antitrypsin deficiency" by J Stolk et al which appeared in the December 2003 issue of Thorax on pages 1027-30, the affiliation address for J Stolk, W H Ng and K F Rabe on page 1030 and the correspondence address on page 1027 should have been Department of Pulmonology, Leiden University Medical Center and not Department of Radiology and Divison of Image Processing. The publishers apologise for this error. 